\title{
Efecto del envejecimieto sobre la capacidad reproductiva de Quercus suber en los bosques maduros de espacios protegidos
}

\section{Effect of senescence on the reproductive ability of Quercus suber mature stands in protected areas}

Marta Pardos 1 , Guillermo Madrigal ${ }^{2}$, Juan J Robledo 3 , José Climent ${ }^{4}$, Ricardo Alía 5 , Fernando Montes ${ }^{6}$, J. Julio Camarero ${ }^{7}$, Álvaro Rubio-Cuadrado ${ }^{8}$, Rafael Calama ${ }^{9}$

${ }^{1,2,9}$ Departamento de Dinámica y Gestión Forestal, INIA-CIFOR, iuFOR, crtra A Coruña Km 7,5, 2040 Madrid; IUFor.

3,4,5 Departamento de Ecología y Genética Forestal, INIA-CIFOR, iuFOR, crtra A Coruña Km 7,5, 2040 Madrid; IUFor

${ }^{7}$ Instituto Pirenaico de Ecología (IPE-CSIC), Avda. Montañana 1005, 50192 Zaragoza,

${ }^{8}$ Departamento de Sistemas y Recursos Naturales, Escuela Técnica Superior de Ingeniería de Montes, Forestal y del Medio Natural, Universidad Politécnica de Madrid. Ciudad Universitaria s/n, 28040, Madrid 


\title{
Resumen
}

La disponibilidad de semilla y su viabilidad son procesos clave para la persistencia de los bosques maduros. Existen evidencias de que la senescencia en los árboles no disminuye la fecundidad en edades avanzadas, pero se desconoce si tiene efectos sobre la viabilidad de la progenie. Este trabajo analiza la influencia de la edad del árbol sobre los atributos biométricos de la semilla, la germinación y la emergencia en los bosques maduros de Quercus suber en los Parques Nacionales de Cabañeros y Monfragüe. En cada uno de los PPNN se seleccionaron 45-48 individuos de Quercus suber, entre jóvenes y senescentes, cubriéndose el rango de diámetros encontrados (24 a $100 \mathrm{~cm}$ en el PN de Cabañeros y 24 a $176 \mathrm{~cm}$ en el PN de Monfragüe). Se tomaron sus coordenadas y se midió su tamaño (diámetro, altura y diámetros de copa). Además, se estimó la edad a $1.30 \mathrm{~m}$ de altura (DBH) mediante la extracción de testigos de madera. En diciembre de 2018 se recogió toda la semilla presente en los individuos seleccionados, que no fue muy abundante. Se determinaron los caracteres biométricos de cada bellota (peso fresco, diámetro, volumen y longitud), analizándose el efecto de la edad del árbol, a través del DBH. Los resultados muestran el efecto del PN, la edad del árbol y el peso de la bellota, tanto en la producción de semilla, como en su germinación y emergencia, aunque la baja cosecha registrada en 2018 enmascara las diferencias esperadas.

Palabras clave: atributos biométricos, emergencia, germinación, senescencia, viabilidad bellota, vecería.

\begin{abstract}
Seed availability and viability are key processes for the persistence of mature forests. There are evidences that tree senescence does not decrease seed fecundity. However, the effect of senescence on the viability of the progeny in mature forests has not been assessed. This study analyses the influence of tree age on biometric traits of the acorn, germination and emergence in mature forests of Quercus suber in Cabañeros and Monfragüe National Parks. We selected 45 to 48 trees in each National Park (NP), covering from young to old trees along the range of diameter at breast height (DBH) found (from 24 to $100 \mathrm{~cm}$ in Cabañeros NP, and from 24 to $176 \mathrm{~cm}$ in Monfragüe NP). Measurements included coordinates, DBH, height and two perpendicular crown diameters. Tree age was estimated at $1.3 \mathrm{~m}$ by extracting cores. In December 2018 seeds were collected from the selected trees. The yield was not abundant due to the marked masting year. After numbering the acorns individually, different biometric traits were measured (fresh weight, diameter, volume and length), and the effect of tree age was analysed, considering DBH as a proxy of tree age. Acorns were seeded to monitor germination and emergence. The results show the effect of site, tree age and acorn size on seed production, biometric traits, germination and emergence. Acorn size varied with parental trees and within a tree. The low yield in 2018 could in part mask the results.
\end{abstract}

Keywords: biometric traits, emergence, germination, senescence, acorn viability, masting habit.

\section{Lista de abreviaturas}

A: tasa máxima de emergencia DBH: diámetro a $1.30 \mathrm{~m}(\mathrm{~cm})$

Dcopa: diámetro de copa $(\mathrm{m})$

G: emergencia acumulada a lo largo del tiempo

$\mathrm{H}$ : altura total $(\mathrm{m})$ $\mathrm{h}(\mathrm{t})$ : probabilidad diaria de emergencia no. bell $\mathrm{m}^{-2}$ : número de bellotas por superficie de copa no. bell: número de bellotas recogidas por árbol $\pi$ : probabilidad de emergencia de una bellota al final de periodo de estudio 


\section{Introducción}

En Europa, y particularmente en los países mediterráneos, la larga historia de uso y gestión de los bosques ha modelado el paisaje y la estructura de los mismos (Grove y Rackham, 2001). Como consecuencia de esta gestión, es difícil encontrar bosques en los que se haya llegado a las últimas fases de envejecimiento y, en cualquier caso, apenas conocemos el grado de madurez de esos bosques (Peterken, 1996). Sin embargo, ante los nuevos paradigmas de multifuncionalidad en la gestión y gestión próxima a la naturaleza, y bajo los nuevos escenarios de cambio global, resulta de gran interés el estudio de las masas envejecidas poco gestionadas (Alejano et al., 2019). En este sentido, los espacios protegidos, y en concreto, los Parques Nacionales, proporcionan un escenario de estudio privilegiado, ya que tienen como objetivo la conservación de los ecosistemas forestales que albergan. La declaración de un Parque Nacional conlleva el cese de la explotación intensiva de sus bosques, de tal forma que a partir de ese momento estos bosques evolucionan y envejecen, dando lugar a cambios en la estructura y dinámica en función de las diferentes estrategias de cada especie. De tal forma, que los bosques maduros que se han conservado en los Parques Nacionales son esenciales para obtener de ellos información que apoye criterios de gestión que imiten la dinámica natural.

Los bosques maduros se caracterizan por albergar una elevada biodiversidad (Lindenmayer y Franklin, 2002), reflejan el efecto de las perturbaciones en forma de legados ecológicos como la acumulación de madera muerta (Franklin y Spies, 1991; Frelich y Reich, 2003; Wirth et al., 2009), son sumideros de carbono importantes a largo plazo (Carey et al., 2001) y proporcionan numerosos servicios ecosistémicos (Nadrowski et al., 2010). La conservación y mantenimiento de los bosques maduros, $\mathrm{y}$, por tanto, su gestión forestal sostenible, dependen en gran medida de su regeneración (Calama et al., 2017). En este sentido, tanto la producción de semilla, como su germinación juegan un papel fundamental en la consecución de la regeneración (Lucas-Borja et al., 2017). Sin embargo, hay poca información sobre la capacidad de los bosques maduros para mantener su capacidad reproductiva y producir suficiente semilla viable a medida que se aproximan a la senescencia (Alejano et al., 2019).

Por tanto, la disponibilidad de semilla y su viabilidad son procesos clave para la persistencia de los bosques maduros. A este respecto, existen evidencias de que la senescencia en los árboles no disminuye la fecundidad en edades avanzadas en términos de producción de semilla (Petit y Hampe, 2006; Mukassabi et al., 2012; Alejano et al., 2019). Sin embargo no se ha estudiado de forma sistemática si la senescencia tiene efectos sobre la viabilidad de la progenie. Podría haber efectos maternales asociados a la edad que reduzcan la germinación de las semillas y la viabilidad de las plántulas en fases tempranas, que son críticas para la regeneración. De esta forma habría senescencia desde el punto de vista de la reproducción efectiva (de la contribución real a la siguiente generación), que es determinante en la eficacia biológica de los individuos, y en definitiva, en la estructura y diversidad futura del bosque.

Además, el tamaño de la semilla también juega un papel importante en el éxito reproductivo, la dinámica poblacional y la estructura del bosque, estableciéndose un 
compromiso entre los beneficios de producir pocas semillas de gran tamaño o muchas de menor tamaño (Smith y Fretwell, 1974). En concreto, en las especies de Quercus, la semilla grande con alto contenido energético presenta una ventaja de dispersión importante (Bartlow et al., 2018). Para las especies de Quercus es además conocida la gran variabilidad en el tamaño de la semilla entre poblaciones (GarcíaNogales et al., 2016), incluso entre individuos de una misma especie (Merouani et al., 2001; Xiao et al., 2004).

Este trabajo se centra en estudiar la influencia de la edad del árbol sobre los atributos biométricos de la semilla, su viabilidad y su capacidad germinativa y de emergencia, centrándonos en los bosques maduros de Quercus suber en los Parques Nacionales de Cabañeros y Monfragüe, ambos Parques representativos del bosque de Quercus mediterráneos. Nuestra hipótesis es que habrá diferencias en las tasas de germinación y emergencia, asociados a la edad del árbol, al tamaño de semilla, al propio individuo, así como al origen las poblaciones, con un fuerte componente estacional, asociado a la vecería de la especie.

\section{Material y métodos}

Para el ensayo se emplearon bellotas de alcornoque (Quercus suber L.), recogidas en los Parques Nacionales (PPNN) de Cabañeros (temperatura media anual $11.5^{\circ} \mathrm{C}$, precipitación anual $645 \mathrm{~mm}$ ) y Monfragüe (temperatura media anual 15.8 ${ }^{\circ} \mathrm{C}$, precipitación anual $635 \mathrm{~mm}$ ). En noviembre de 2018, previo a la recogida de las bellotas, se realizó un conteo visual de la cosecha presente en la copa de los árboles. La escasa cosecha de bellotas registrada por la especie en 2018 limitó el número de árboles seleccionados a lo largo del rango de diámetros disponibles a 48 árboles en el PN de Cabañeros $\left(4^{\circ} 30^{\prime} 5^{\prime}\right.$ 'W, $\left.39^{\circ} 22^{\prime} 47^{\prime} \mathrm{N}\right)$ y 45 árboles en el PN de

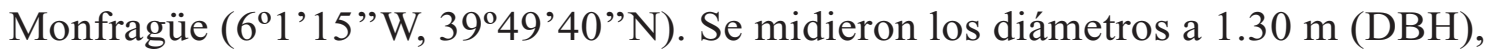
la altura total $(\mathrm{H})$, los diámetros de copa en dos direcciones perpendiculares (Dcopa) y sus coordenadas, estableciéndose cuatro rangos de clases diamétricas (Tabla 1). Mientras que en el PN de Monfragüe se cubrió todo el rango de diámetros disponible (de 24 a más de $100 \mathrm{~cm}$ ), en el PN de Cabañeros la mayor parte de los árboles con cosecha visible se encontraron en el rango diamétrico entre 24 y 75 $\mathrm{cm}$, localizándose sólo un árbol de $100 \mathrm{~cm}$ de diámetro con semilla.

En la Tabla 1 se muestra el número de árboles muestreados por clase diamétrica dentro de cada PN. Además, se extrajeron dos testigos de madera a $1.3 \mathrm{~m}$ de altura usando barrenas Pressler para estimar la edad a dicha altura. Después del secado, montaje y lijado de los testigos se contaron los anillos de crecimiento con LINTAB y el software TSAP (Frank Rinn, Heidelberg, Alemania). Asumiéndose una sección circular del tronco, se estimó el número de anillos ausentes en aquellos testigos que no alcanzaron la médula o en aquellos árboles con el centro podrido. La estimación de la edad teórica de estos árboles se realizó ajustando un modelo exponencial diámetro-edad, restando los crecimientos acumulados del diámetro bajo corcho medido en campo para transformar los crecimientos presentes en 
la muestra en diámetros. La estimación de la edad en la mitad de los árboles muestreados en cada PN muestra una buena correlación entre la edad del árbol y su DBH (Figura 1), habiéndose estimado una edad máxima de los árboles entorno a los 200 años en el PN de Monfragüe y hasta de 300 años en el PN de Cabañeros.

Tabla 1. Variables dasométricas (diámetro normal: $\mathrm{DBH}$, altura: $\mathrm{H}$, diámetro de copa: Dcopa), número de bellotas por superficie de copa (no. bell. $\mathrm{m}^{-2}$ ) (medias \pm error estándar), número de árboles seleccionados (no. arb.) y rango del número de bellotas recogidas por árbol (no. bell.) por rango de clase diamétrica en cada PN.

\begin{tabular}{|c|c|c|c|c|c|c|}
\hline PN Cabañeros & DBH (cm) & $\overline{\mathrm{H}(\mathrm{cm})}$ & $\overline{\text { Dcopa (m) }}$ & no. bell. $\mathrm{m}^{-2}$ & no. arb. & no. bell. \\
\hline $24<$ DBH $1<50$ & $5.3 \pm 1.1$ & $9.1 \pm 0.3$ & $6.1 \pm 0.2$ & $7.5 \pm 1.6$ & 30 & $10-161$ \\
\hline $50<$ DBH $2<75$ & $63.4 \pm 1.8$ & $11.2 \pm 0.5$ & $9.2 \pm 0.4$ & $3.8 \pm 0.9$ & 17 & $9-88$ \\
\hline $75<$ DBH3 $<100$ & 100 & 12.2 & 13.7 & 1.3 & 1 & 11 \\
\hline \multicolumn{7}{|l|}{ PN Monfragüe } \\
\hline $24<$ DBH1 $<50$ & $41.3 \pm 3.0$ & $8.3 \pm 0.5$ & $8.6 \pm 0.7$ & $6.1 \pm 0.3$ & 15 & $10-64$ \\
\hline $50<$ DBH $2<75$ & $63.6 \pm 2.6$ & $10.1 \pm 0.3$ & $12.6 \pm 0.6$ & $7.8 \pm 0.2$ & 13 & $9-160$ \\
\hline $75<$ DBH3 $<100$ & $87.7 \pm 2.2$ & $12.2 \pm 0.6$ & $14.3 \pm 0.6$ & $8.4 \pm 0.2$ & 12 & $19-131$ \\
\hline DBH4>100 & $128.5 \pm 12.5$ & $11.5 \pm 0.7$ & $13.7 \pm 0.7$ & $8.2 \pm 0.5$ & 5 & $14-138$ \\
\hline
\end{tabular}

En diciembre de 2018 se recogió toda la semilla presente en los individuos seleccionados, que, como se ha comentado anteriormente, no fue abundante al acusarse el carácter vecero de la especie. A partir de estos datos se calculó para cada árbol el número de bellotas recogidas por $\mathrm{m}^{2}$ de copa. Se recogieron 1268 bellotas en el PN de Cabañeros y 1991 en el PN de Monfragüe (Tabla 1).

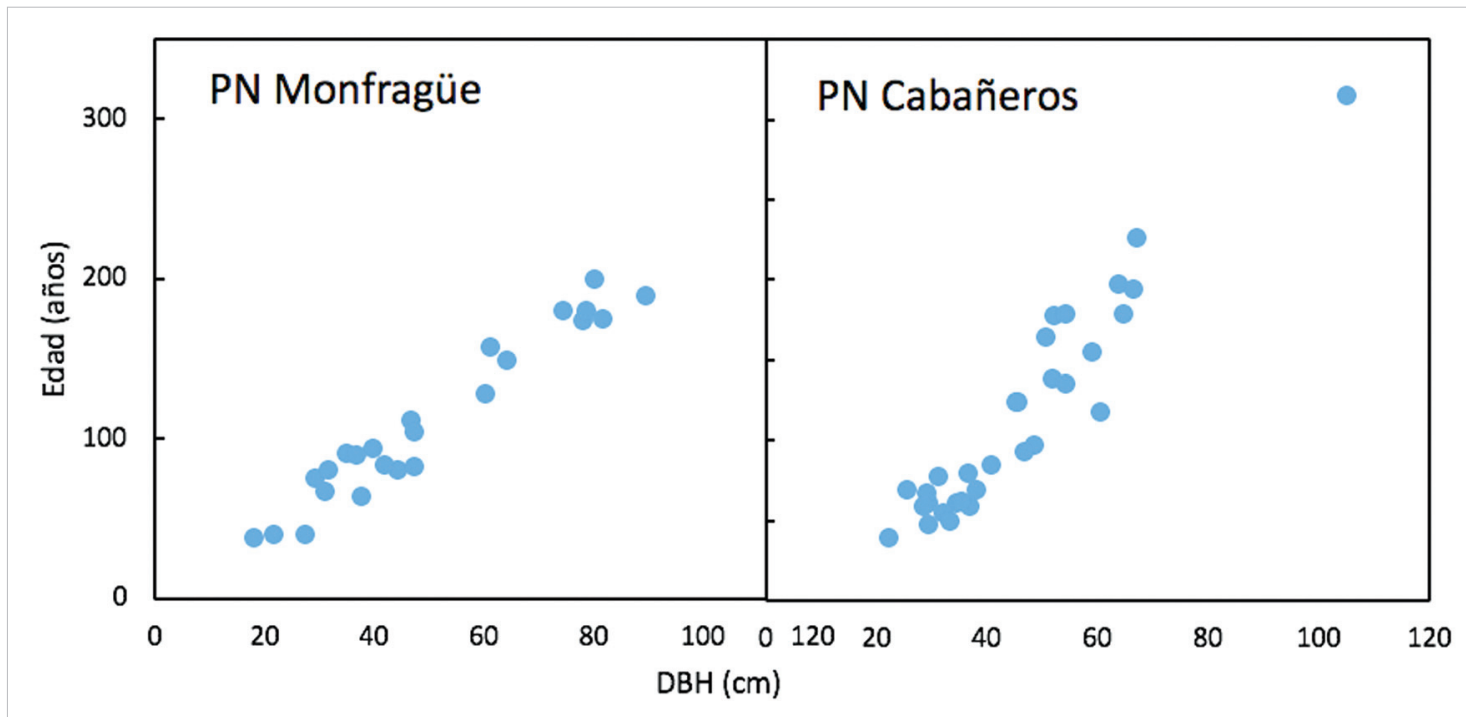

Figura 1. Relación entre la edad de los árboles (estimada a partir de los testigos de madera extraídos) y el DBH, para los árboles muestreados en los PPNN de Cabañeros y Monfragüe. 
A mediados de enero de 2019, las bellotas se numeraron individualmente, se determinaron los caracteres biométricos de cada bellota (peso fresco, diámetro y longitud) y se calculó su volumen, asimilándolo al de un elipsoide. Inmediatamente después, las bellotas se pusieron a germinar en bandejas de plástico con perlita humedecida, bajo condiciones controladas de humedad y temperatura, realizándose el conteo semanal de la germinación durante dos meses. Se calculó el porcentaje de germinación diario, así como el acumulado.

Transcurridos dos meses de seguimiento de la germinación se trasplantaron el $50 \%$ de las bellotas a bandejas Forest-Pot 300 de 50 alveolos, rellenas con una mezcla de turba y vermiculita, según un diseño aleatorizado de fila-columna latinizado, con 10 bloques incompletos y una unidad experimental de 3 semillas teniendo en cuenta la familia (es decir, el árbol) y el parque nacional. Las bandejas se mantuvieron en un invernadero, con control de temperatura y riego (4 días a la semana). El número de bellotas trasplantadas fue de 9,18 ó 30, según la familia. Se realizó el seguimiento una vez por semana de la emergencia hasta mediados de mayo (durante un total de 80 días), calculándose la emergencia diaria y acumulada.

Basándose en las correlaciones obtenidas entre la edad de los árboles y el DBH (Figura 1), todos los análisis estadísticos se realizaron considerando el efecto del DBH o del rango diamétrico. Para cada PPNN se determinó el efecto de la clase diamétrica y los efectos inter e intra-familia (es decir, el árbol) sobre los caracteres biométricos de la bellota (peso fresco, diámetro, longitud y volumen), el número de bellotas por $\mathrm{m}^{2}$ de copa, la germinación y la emergencia, mediante ANOVA y test de Tukey de comparación de medias. Se calcularon también las correlaciones (test de correlación de Pearson) entre los caracteres biométricos de las bellotas y los porcentajes de germinación y emergencia acumulados.

La emergencia de las plántulas se estudió a dos escalas diferentes, el árbol y la bellota. A nivel de árbol, la curva de tasa de emergencia acumulada (G) para las bellotas de un árbol (porcentaje de bellotas emergidas en un tiempo $t$ ) se ajustó mediante una función logística (Torres y Frutos, 1989):

$$
\boldsymbol{G}=\frac{\boldsymbol{A}}{1+e^{(\beta-k t)}}
$$

donde $\boldsymbol{A}$ (la tasa máxima de emergencia), $\beta$ y k son parámetros desconocidos a estimar en el ajuste. Para considerar el efecto del tamaño del árbol (y por consiguiente de la edad) sobre la curva de emergencia a escala de árbol se propone expandir los parámetros $\boldsymbol{A}, \beta \mathrm{y}$ k de manera independiente, en función del diámetro $(\mathrm{DBH})$ del árbol:

$$
\begin{aligned}
& A=a+a_{1} * D B H \\
& \beta=b+b_{1} * D B H \\
& k=c+c_{1} * D B H
\end{aligned}
$$

En una primera fase se analizará la expansión por separado de cada uno de los parámetros, para posteriormente estudiar la expansión simultánea de dos de ellos 
y por último la de los tres parámetros. La comparación entre los modelos con distinto número de parámetros se realiza mediante el test $F$ extra de la suma de cuadrados no lineal (Pillsbury y McDonald (1995). En este test, siendo el modelo reducido el de menor número de parámetros, y el completo el de mayor número de parámetros, bajo la hipótesis nula de que el modelo completo no se comporta mejor que el modelo reducido, $\mathrm{F}$ se distribuye de acuerdo a una distribución de FisherSnedecor.

$$
\begin{aligned}
& \frac{\text { SSRred }- \text { SSRcompleto }}{\text { dfred }- \text { dfcompleto }} \\
& \mathrm{F}=
\end{aligned}
$$$$
\approx \text { Ffisher (1- } \alpha \text {; dfred-dfcompleto; dfcompleto) }
$$

\section{SSRcompleto dfcompleto}

donde donde SSRred y SSRcompleto son la suma de cuadrados del error del modelo reducido y completo; $d$ fred y dfcompleto se refieren a los grados de libertad del error de los modelos reducido y completo, respectivamente.

Dado el interés en estudiar el posible efecto del tamaño sobre los parámetros que definen el modelo no lineal de emergencia acumulada, y al objeto de evitar la sobreparametrización del modelo, se procedió a realizar los análisis por separado para cada uno de los parques. Asimismo, se evaluó la inclusión de un efecto aleatorio de árbol madre, que resultó no significativo. A escala de la bellota se estudiaron dos aspectos diferentes al objeto de determinar:

1. Qué factores influyen en la probabilidad de que la bellota haya emergido en plántula al final del estudio.

2. Factores que influyen en el tiempo medio de emergencia de una bellota. D

Como posibles variables explicativas se consideraron el diámetro a $1.30 \mathrm{~m}$ de altura del árbol madre, el origen de la bellota (es decir, el Parque Nacional) y atributos biométricos de la bellota. Dada la alta multicolinealidad detectada entre la longitud, el diámetro, el volumen y el peso fresco de la bellota se seleccionó esta última variable, al ser la que presentaba mejor bondad del ajuste en los modelos. El bajo valor del factor de inflación de la varianza observado entre DBH y peso fresco $(\mathrm{VIF}=1.25)$ justifica el que pese haber identificado correlación entre ambas variables se considere la inclusión de las mismas en el modelo.

Para calcular la probabilidad $(\pi)$ de emergencia de una bellota al final de periodo de estudio ( 80 días) se empleó un modelo mixto lineal generalizado $(\mathrm{McCu}-$ llagh y Nelder, 1989):

$$
\pi=\frac{e^{x \beta+u}}{1+e^{x \beta+u}}
$$


donde $\beta$ es el vector de parámetros a estimar, que indica el conjunto de potenciales variables explicativas y u representa un efecto aleatorio del árbol madre (familia).

Por último, el tiempo de emergencia de una bellota se modelizó a través de modelos de análisis de vida mediante una función de proporción de riesgo (Lawless, 2003). La probabilidad de que la planta nazca en un tiempo $t(E(t))$ se define a través de la suma de las probabilidades diarias de emergencia:

$$
\mathrm{E}(\mathrm{t})=1-\exp \left(-\sum_{\mathrm{t}=1}^{\mathrm{T}} h(t)\right)
$$

donde $\mathrm{h}(\mathrm{t})$ se denomina función de riesgo (hazard fucntion), y representa la probabilidad diaria de emergencia para un día t cualquiera. La función de riesgo se va a relacionar linealmente con las variables diámetro del árbol madre, parque de origen y peso fresco de la bellota.

Todos los análisis estadísticos se realizaron usando el paquete estadístico SAS 9.2 (SAS Institute, Cary, NC, USA).

\section{Resultados}

Los resultados de los caracteres biométricos de las bellotas mostraron en ambos PPNN gran variabilidad entre árboles (familias, $\mathrm{P}$-valor $<0.0001$ ) y dentro de un individuo (P-valor $<0.0001$ ), con independencia de la clase diamétrica. El peso medio de las bellotas fue de $3.2 \mathrm{~g}$ en el PN Cabañeros, oscilando entre 2.5 y $4.5 \mathrm{~g}$, según clase diamétrica, en el PN Monfragüe. Se registró también poca variación entre clases diamétricas en el volumen medio de la bellota de los árboles del PN Cabañeros $\left(28-30 \mathrm{~cm}^{3}\right)$, mientras que en el PN Monfragüe osciló entre 24.3 y $39.2 \mathrm{~cm}^{3}$. Además, mientras que no se observaron diferencias significativas en los rangos biométricos de las bellotas entre las dos clases diamétricas en el PN de Cabañeros, sí que se registraron diferencias en el PN de Monfragüe, con valores significativamente mayores de diámetro ( $\mathrm{P}$-valor $=0.056)$, longitud $(\mathrm{P}$-valor $=0.0267)$, volumen $(\mathrm{P}$-valor $=0.0024)$ y peso fresco de bellota $(\mathrm{P}$-valor $=0.0061)$ en los árboles más gruesos (Tabla 2). Además, en el PN Monfragüe se registró un efecto significativo de la clase diamétrica en el número de bellotas por superficie de copa (Pvalor $<0.0001$ ).

Transcurridos dos meses, los porcentajes de germinación acumulada fueron de $27.3 \%$ en el PN de Cabañeros y de $43.2 \%$ en el PN de Monfragüe, sin que hubiera un efecto de la clase diamétrica, pero con una gran variabilidad entre familias (Pvalor $<0.0001$ ). Los porcentajes de emergencia acumulada al final del ensayo ( 80 días) fueron de $47.2 \%$ en el PN de Cabañeros y de $63.9 \%$ en el PN de Monfragüe (Figura 2), también de nuevo con gran variabilidad entre familias $(\mathrm{P}-\mathrm{valor}<0.0001$ ) y sin diferencias significativas entre clases diamétricas. El análisis de correlación entre los parámetros biométricos, el número de bellotas por superficie de copa y los porcentajes de germinación y emergencia acumulados finales mostró, para ambos 
Tabla 2. Resultados del ANOVA y valores medios ( \pm error estándar) de los caracteres biométricos de las bellotas de alcornoque por rango de diámetro (DBH; ver clases diamétricas en Tabla 1), medidos en los PPNN de Cabañeros y Monfragüe. Las letras distintas muestran los efectos significativos $(\mathrm{P}<0.05)$ del rango de DBH sobre el carácter correspondiente.

\begin{tabular}{|c|c|c|c|c|}
\hline & $\begin{array}{c}\text { Peso fresco } \\
(\mathrm{g})\end{array}$ & $\begin{array}{c}\text { Diámetro } \\
(\mathrm{mm})\end{array}$ & $\begin{array}{c}\text { Longitud } \\
(\mathrm{mm})\end{array}$ & $\begin{array}{c}\text { Volumen } \\
\left(\mathrm{cm}^{3}\right)\end{array}$ \\
\hline \multicolumn{5}{|l|}{ PN Cabañeros } \\
\hline $24<$ DBH1 $<50$ & $3.26 \pm 0.27^{\mathrm{a}}$ & $13.49 \pm 0.35^{\mathrm{a}}$ & $27.98 \pm 1.00 \mathrm{a}$ & $27.99 \pm 2.03^{\mathrm{a}}$ \\
\hline $50<$ DBH $2<75$ & $3.23 \pm 0.38 \mathrm{a}$ & $13.85 \pm 0.3^{\mathrm{a}}$ & $27.44 \pm 1.54^{\mathrm{a}}$ & $29.63 \pm 3.15^{\mathrm{a}}$ \\
\hline \multicolumn{5}{|l|}{ PN Monfragüe } \\
\hline $24<$ DBH $1<50$ & $2.55 \pm 0.38 \mathrm{c}$ & $13.06 \pm 0.56 b$ & $26.01 \pm 1.44 b$ & $24.31 \pm 3.12 \mathrm{c}$ \\
\hline $50<$ DBH $2<75$ & $2.91 \pm 0.37 \mathrm{bc}$ & $13.00 \pm 0.55 b$ & $26.84 \pm 1.28 \mathrm{ba}$ & $24.35 \pm 2.72 \mathrm{c}$ \\
\hline $75<$ DBH3 $<100$ & $3.36 \pm 0.42 b$ & $13.92 \pm 0.57^{\mathrm{a}}$ & $27.56 \pm 1.33^{\mathrm{a}}$ & $29.38 \pm 3.38 b$ \\
\hline DBH4>100 & $4.51 \pm 0.47 \mathrm{a}$ & $15.11 \pm 0.45^{\mathrm{a}}$ & $31.56 \pm 1.35^{\mathrm{a}}$ & $39.22 \pm 3.69 a$ \\
\hline
\end{tabular}

Parques, una correlación positiva con el número de bellotas por superficie de copa $(\mathrm{r}=0.60503$; P-valor $<0.0001)$ y con el peso fresco de la bellota $(\mathrm{r}=0.30546$; $\mathrm{P}$ valor $=0.0368$ ).

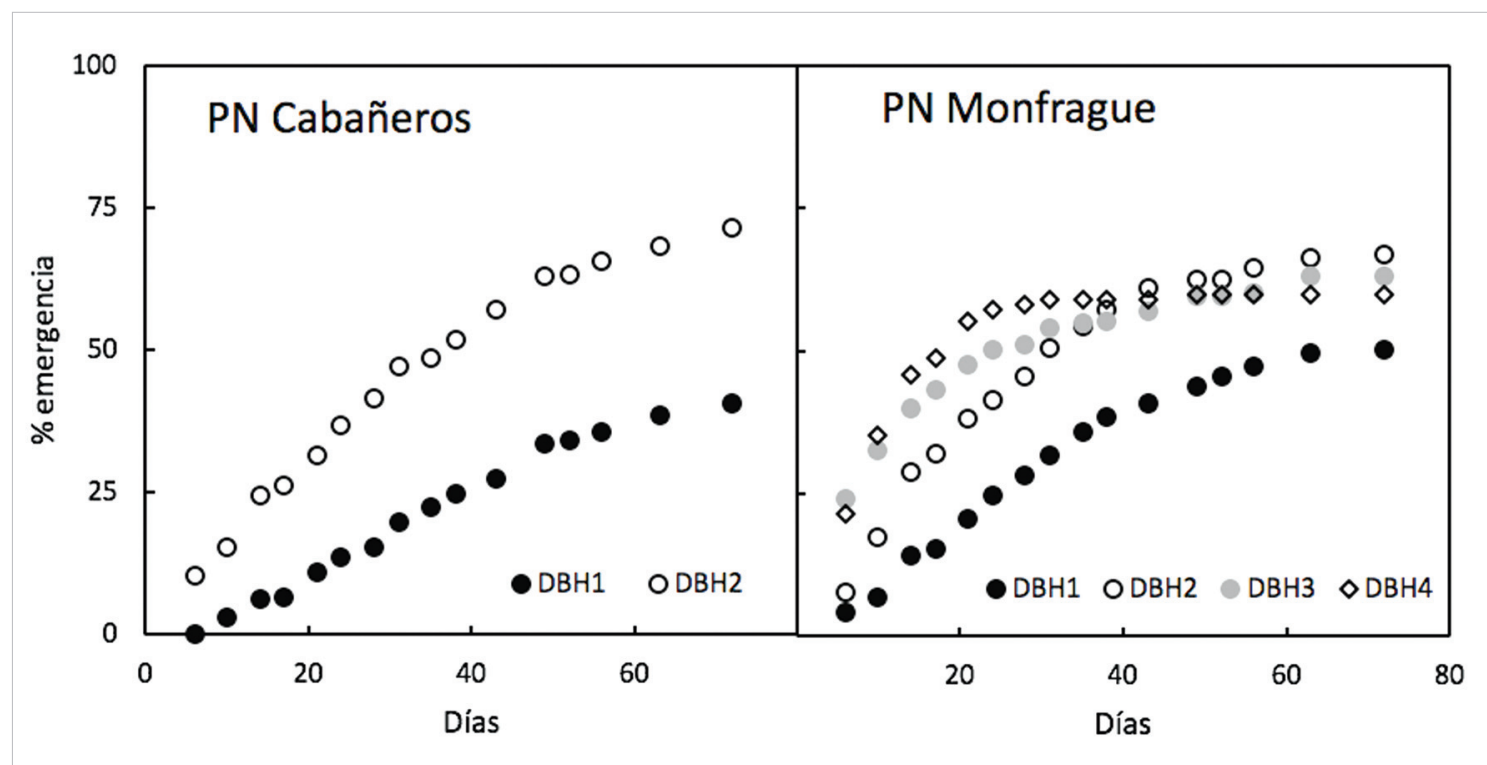

Figura 2. Porcentaje de emergencia acumulada de bellotas de alcornoque a lo largo de tiempo según clase diamétrica (DBH1, DBH2, DBH3 y DBH4) en los PPNN de Cabañeros y Monfragüe.

Los resultados del ajuste de la tasa de emergencia acumulada por árbol a lo largo del tiempo (G) para cada PN a una función logística y la expansión de los parámetros de la curva se muestran en las Tabla 3 y 4 . La posterior aplicación del test $F$ extra de la suma de cuadrados no lineal refleja que para ambos PPNN el mejor modelo es 
aquel en el que los parámetros $a$ y $b$ se expanden en función del diámetro (DBH) del árbol, apuntando a un efecto del tamaño del árbol sobre la velocidad de emergencia. En cualquier caso, el hecho de que el parámetro de expansión b1 sea no significativo en el PN Cabañeros nos indica que en este Parque el diámetro tiene una mayor influencia sobre la tasa final de emergencia, mientras que en el PN Monfragüe está afectando también al punto de inflexión de la curva, tal y como se recoge en la Figura 2.

Tabla 3. Comparación de los distintos modelos de emergencia acumulada a lo largo del tiempo con expansión de parámetros en función del diámetro (DBH) del árbol, para los dos Parques Nacionales.

\begin{tabular}{|c|ccc|ccc|}
\hline & \multicolumn{3}{|c|}{ CABANEROS } & \multicolumn{3}{c|}{ MONFRAGÜE } \\
\hline Parámetros expandidos & SSR & df & p-valor F test & SSR & df & p-valor F test \\
\hline Ninguno & 416882 & 492 & $<0.0001$ & 771083 & 582 & $<0.0001$ \\
"a" & 384860 & 491 & 0,0055 & 731940 & 581 & 0,0003 \\
"b" & 386201 & 491 & 0,0011 & 727667 & 581 & 0,0078 \\
"c" & 390179 & 491 & $<0.0001$ & 726487 & 581 & 0,0195 \\
"a y b" & 380703 & 490 & 0,9859 & 721485 & 580 & 0,3573 \\
\hline "a y c" & 382030 & 490 & 0,1820 & 722562 & 580 & 0,1523 \\
"b y c" & 385972 & 490 & 0,0010 & 723105 & 580 & 0,0993 \\
\hline "a, b y c" & 380692 & 489 & - & 720198 & 579 & - \\
\hline
\end{tabular}

Abreviaturas: SSR: suma cuadrados de los residuos; df, grados de libertad. En el F-test se compara cada modelo con el modelo con menor SSR de la siguiente categoría (p.ej. los modelos con un parámetro expandido se comparan con el modelo con los parámetros "a" y "b" expandidos).

Tabla 4. Resultado del ajuste del modelo para la tasa de emergencia G asumiendo expansión de los parámetros a y b.

\begin{tabular}{|c|cc|cc|}
\hline & \multicolumn{2}{|c|}{ CABANEROS } & \multicolumn{2}{c|}{ MONFRAGUE } \\
\hline Parámetros & Estimador & p-valor & Estimador & p-valor \\
\hline a & 27,1535 & 0,0094 & 44,162 & $<0.0001$ \\
a1 & 0,5185 & 0,0022 & 0,2033 & 0,0078 \\
b & 2,9932 & $<0.0001$ & 2,515 & 0,0005 \\
b1 & $-0,0189$ & 0,0779 & $-0,0193$ & 0,0144 \\
c & 0,0866 & $<0.0001$ & 0,1054 & $<0.0001$ \\
\hline
\end{tabular}

Tabla 5. Resultados del modelo mixto lineal generalizado sobre la probabilidad de emergencia al final del periodo de estudio.

\begin{tabular}{|c|c|c|}
\hline & Estimador & P-valor \\
\hline Constante & 0.4442 & 0.0008 \\
\hline DBH & 0.00115 & 0.4566 \\
\hline Peso fresco bellota & 0.05052 & $<0.0001$ \\
\hline PN Cabañeros & -0.1924 & 0.0173 \\
\hline PN Monfragüe & 0 & - \\
\hline
\end{tabular}

Los resultados de la probabilidad $(\pi)$ de emergencia de una bellota al final de periodo de estudio se recogen en la Tabla 5. Se observa una menor probabilidad de emergencia en el PN de Cabañeros. En ambos PPNN dicha probabilidad aumenta con 
el peso fresco de la bellota, sin que se observe un efecto directo del diámetro (DBH) del árbol.

Se observa también un efecto muy significativo del efecto aleatorio del árbol madre, registrándose algunas familias con una probabilidad de emergencia superior a la media no atribuible al tamaño del mismo (Figura 3).

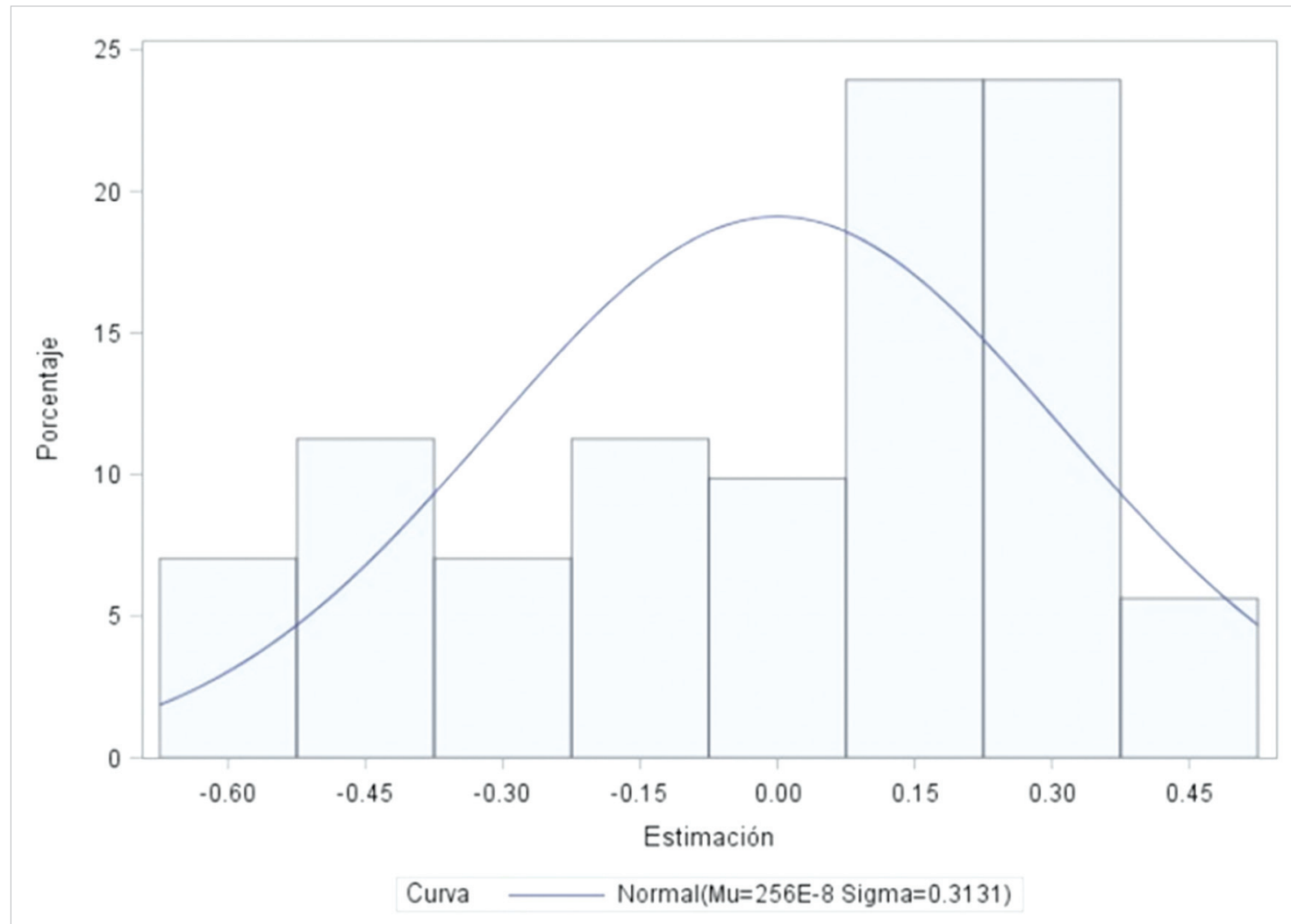

Figura 3. Histograma de frecuencias para el efecto aleatorio de árbol madre (familia) asociado al modelo de probabilidad de emergencia, una vez descontados los efectos del peso fresco de bellota, el DBH y el Parque Nacional.

Los resultados de la probabilidad de que la planta haya emergido en un tiempo $t$ (Tabla 6) muestran una emergencia más temprana en las bellotas con mayor peso fresco. Además, las bellotas procedentes de árboles de mayor diámetro presentan una emergencia más temprana. Por último, las bellotas del PN de Monfragüe emergen antes que las del PN de Cabañeros.

Tabla 6. Parámetros del modelo de análisis de vida para probabilidad de emergencia de una planta en un tiempo t.

\begin{tabular}{|c|c|c|}
\hline & Estimador & P-valor \\
\hline DBH & 0.00380 & $<0.0001$ \\
\hline Peso fresco bellota & 0.19056 & $<0.0001$ \\
\hline PN Cabañeros & -0.43353 & $<0.0001$ \\
\hline PN Monfragüe & 0 & - \\
\hline
\end{tabular}




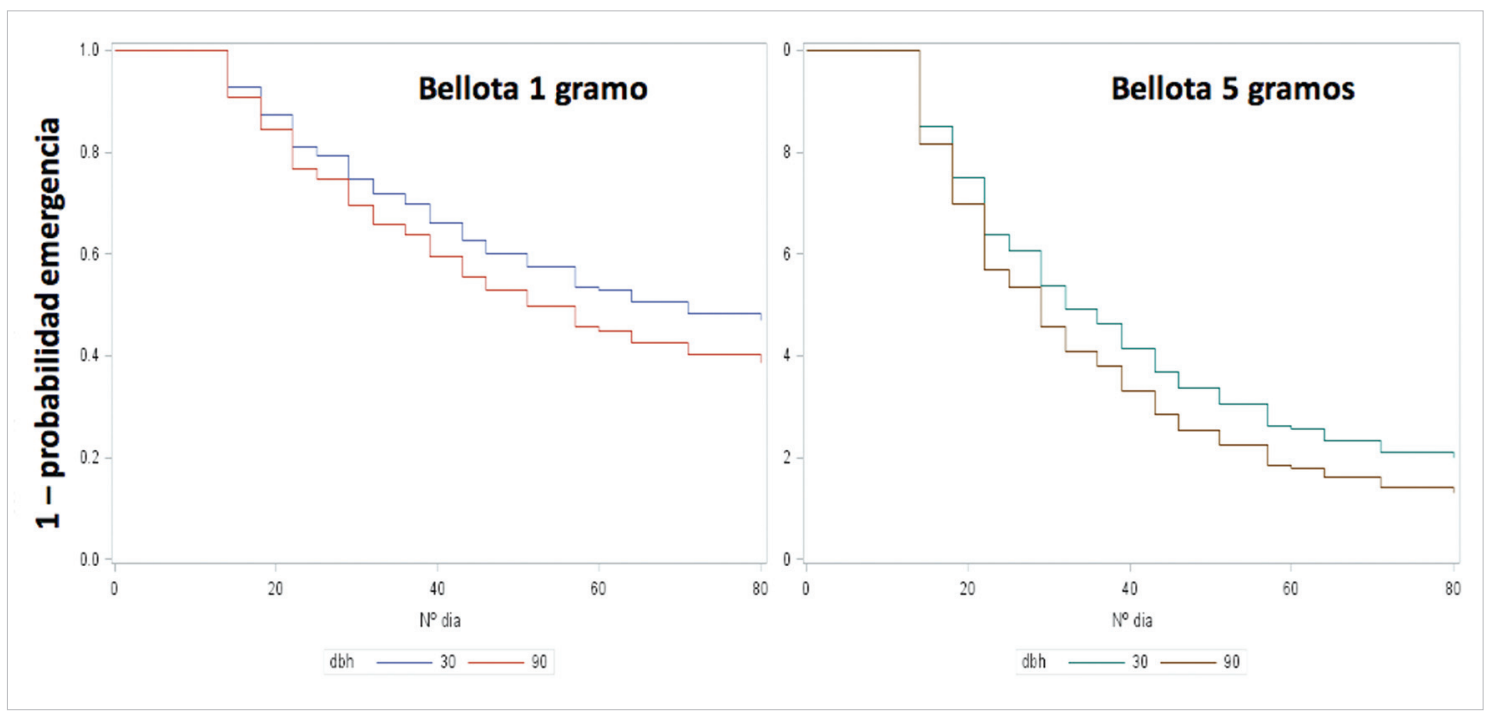

Figura 4. muestra cómo el tiempo necesario para alcanzar una probabilidad de emergencia del 50\% en las bellotas del PN Monfragüe se reduce de 50 (para un DBH de $90 \mathrm{~cm}$ ) y 65 días (para un DBH de $30 \mathrm{~cm}$ ) a 30 (para un DBH de $90 \mathrm{~cm}$ ) y 35 días (para un DBH de $30 \mathrm{~cm}$ ) al aumentar el peso fresco de la bellota de 1 (panel de la izquierda) a $5 \mathrm{~g}$ (panel de la derecha).

\section{Discusión}

La regeneración es un proceso clave para la persistencia de los bosques maduros (Oliver y Larson, 1990), considerándose como un proceso que conlleva múltiples etapas consecutivas, que normalmente se identifican como umbrales sucesivos de supervivencia de las plántulas (Pukkala y Kolström, 1992). Entre estas etapas, la disponibilidad y viabilidad de la semilla juegan un papel fundamental. En este estudio se ha identificado el papel que el tamaño de bellota y su relación con el tamaño y la edad del árbol juegan sobre diferentes caracteres biométricos, la germinación y la emergencia, corroborándose, además, la variabilidad entre y dentro de individuos (Merouani et al., 2001) y el importante condicionante que supone la vecería de la especie.

Los resultados de los caracteres biométricos muestran un rango de pesos medios de la bellota de alconorque entre 2.4 y $4.4 \mathrm{~g}$; un valor de longitud media del eje mayor de la bellota de $2.7 \mathrm{~cm}$ y un diámetro medio de la bellota de $1.3 \mathrm{~cm}$. Estos valores resultan bajos si se comparan con el rango medio de pesos medios (entre 6 y $7 \mathrm{~g}$ ), longitud (hasta $5 \mathrm{~cm}$ ) y diámetro $(1.8 \mathrm{~cm}$ ) descritos para la especie (Vieira Natividade, 1991; Montero y Cañellas, 1999). Por ello, las bellotas recogidas en 2018 se corresponderían con bellotas de pequeño tamaño. A esto se une la gran variabilidad encontrada en 2018 en la producción de fruto tanto entre individuos (Tabla 1) como dentro de un mismo individuo, así como la ausencia de cosecha en muchos de ellos y la presencia de bellotas abortadas. Como se ha comentado anteriormente, 2018 fue un año marcadamente no vecero. La limitada cosecha en 2018 se relaciona, en parte, con las condiciones climáticas secas tanto durante el perio- 
do de floración en primavera de 2018, como durante la maduración del fruto ese otoño, viéndose afectado no sólo el alcornoque, sino todas las especies de Quercus presentes en los dos PPNN. Concretamente, en el alcornoque, la vecería conlleva que cada periodo de 10 años, rara vez se cuenten con más de dos o tres cosechas abundantes (Vieira Natividade, 1991). Dicho fenómeno se relaciona con las condiciones climáticas (sequía y helada tempranas), con la estrategia reproductiva de la especie para mejorar su eficiencia de polinización (Petit y Hampe, 2006), e incluso con el patrón de maduración de la bellota (anual $v s$ bianual), que puede ser diferente entre los individuos de una población (Pons y Pausas, 2012).

Las diferencias registradas entre individuos en los caracteres biométricos de la bellota, con independencia del diámetro y edad del árbol, pone de relieve la gran variabilidad intraespecífica en estos caracteres encontradas en los dos PPNN, variabilidad que puede estar más relacionada con aspectos genéticos, fisiológicos y ambientales (Aizen y Patterson, 1990; Aizen y Woodcock, 1992; Long y Jones, 1996), que con la edad o el tamaño del árbol. Además, en el PN de Monfragüe se registró un incremento en los valores de los caracteres biométricos al aumentar el diámetro del árbol, así como un incremento en la cosecha, es decir, bellotas mayores en los árboles de más edad y con tronco más grueso, así como cosechas más abundantes, lo que apoya el hecho de que la senescencia en los árboles no disminuye la fecundidad en edades avanzadas en términos de producción de semilla (Petit y Hampe, 2006; Mukassabi et al., 2012). La ausencia de dicho efecto en el PN Cabañeros puede estar relacionada con la ausencia de cosecha en 2018 en los árboles de mayor diámetro, lo que podría apuntar a una más limitada o incluso nula producción en estos individuos en los años no veceros. En este PN podría existir un sesgo de los datos hacia los árboles de menor diámetro, que sí presentaron producción de bellota en 2018.

Con respecto a los resultados de germinación y emergencia, se obtuvieron en ambos casos valores inferiores a los valores medios registrados para la especie, que son superiores al $80 \%$ (Vieira Natividade, 1991), sin que hubiera un efecto de la clase diamétrica, y con una gran variabilidad entre familias. Cabe mencionar que dichos valores medios de germinación y emergencia descritos para la especie se corresponden con bellotas de tamaño medio. Como se ha comentado anteriormente, las bellotas de este estudio estarían en la clasificación de bellotas pequeñas, y a pesar de ello, sus valores de germinación y emergencia se alejan de los que se pueden obtener para ese tamaño (Merouani et al., 2001).

En ambos PPNN la probabilidad de emergencia aumenta con el peso fresco de la bellota, es decir, las bellotas de más tamaño tienen mayor probabilidad de emergencia. Sin embargo, el efecto del tamaño de la bellota sobre la probabilidad de germinación y emergencia posterior, no es unánime. Así, algunos autores no lo consideran un factor determinante (Xiao et al., 2004), al menos durante los 50 primeros días (ver p.ej. Yi et al., 2015). Hay incluso estudios que demuestran que las bellotas son capaces de germinar cuando han perdido hasta el 50\% del cotiledón (Bartlow et al., 2018), mientras que otros estudios postulan lo contrario (Gómez 2004; Sánchez-Montes de Oca et al., 2018). Estos últimos estudios consideran que el au- 
mento en el peso fresco de la bellota mejora la germinación y posterior emergencia, resultando, por tanto, un carácter importante de la historia vital de los Quercus. En nuestro estudio, la presencia de bellotas de mayor tamaño en el PN de Monfragüe, al menos en los árboles de la clase diamétrica superior, se tradujo en mayores tasas de geminación y emergencia. Dichas diferencias en los porcentajes de emergencia entre los dos PPNN, cuando la bellota se cultiva en invernadero bajo las mismas condiciones, puede reflejar la capacidad de los Quercus para adaptarse a germinar bajo diferentes condiciones ecológicas (Westoby et al., 1992).

Por su parte, cabe destacar la gran variabilidad en la probabilidad de emergencia entre familias y dentro del individuo, similar a la observada en los parámetros biométricos de las bellotas. De hecho, en otros estudios con Quercus la mayor parte de la variabilidad en el tamaño de la bellota se produce dentro del individuo, más que entre individuos (Shi et al., 2019). Esto apunta a un fuerte efecto materno no genético en este carácter, relacionado con la posición de las bellotas en la copa, indicando que la mayor parte de la variación en el tamaño de la bellota es debido a las limitaciones fisiológicas o morfológicas en el reparto de recursos a las mismas dentro de cada individuo y entre ramas (Moles y Leishman, 2008).

En cuanto a los resultados relativos a una emergencia más temprana en las bellotas con mayor peso fresco, este fenómeno se observa en los árboles viejos y de troncos más gruesos, sobre todo en el PN de Monfragüe. La mayor rapidez en la emergencia al aumentar el peso de la semilla se puede atribuir a efectos maternos (Gómez, 2004). Esta circunstancia podría suponer una ventaja adaptativa, pues podrán ocupar antes el espacio disponible bajo las copas, lo que también puede resultar beneficioso en la fase posterior de establecimiento del regenerado por la protección que proporciona el arbolado adulto en las primeras edades (Sánchez-Montes de Oca et al., 2018). Además, un periodo de crecimiento más prolongado antes del comienzo de la sequía estival podría incrementar las posibilidades de soportar la misma (Aranda et al., 2007).

\section{Conclusiones}

En este estudio se pone de manifiesto la influencia del peso de la bellota sobre los caracteres estudiados, de tal forma que se asocia un mayor peso fresco a probabilidades superiores de germinación y de emergencia, así como a una emergencia más temprana. Además, en el PN de Monfragüe las bellotas de mayor peso son producidas por los árboles más viejos y de troncos más gruesos, de tal forma que, al menos para los árboles muestreados, la senescencia de los mismos no disminuye ni la producción de semilla, ni su germinación y posterior emergencia, sino que aumenta la producción de bellotas y también su tamaño, lo que favorece una germinación temprana y emergencia. Nuestros resultados confirman también la gran variabilidad en el peso de la semilla entre individuos e incluso dentro de un mismo individuo, según la posición en las ramas. Por último, la marcada ausencia de bellotas registrada durante 2018 puede producir un sesgo en el análisis de los resul- 
tados para el PN de Cabañeros, al no localizarse árboles de las clases diamétricas superiores (más de $75 \mathrm{~cm}$ de diámetro) con cosecha; ni poderse incluir en el estudio otras especies de Quercus presentes en los dos PPNN. Es importante, por ello, disponer de series más largas de producción de bellota, que proporcionará información interesante sobre la sincronización de la vecería.

\section{Agradecimientos}

Este proyecto cuenta con el apoyo de la Fundación Biodiversidad del Ministerio para la Transición Ecológica. Agradecemos a los directores de los PPNN de Cabañeros (Angel Gómez Manzaneque) y Monfragüe (Angel Rodríguez Martín) el apoyo para la realización del trabajo de campo; así como al personal del INIA, Ana Carmen de la Cruz, Ana Parras, Fernando del Caño, Eduardo Ballesteros y Sergio Sansegundo el apoyo en la instalación del ensayo en el invernadero y en las mediciones de laboratorio.

\section{Bibliografía}

Aizen, M.A.; Patterson, W.A.; 1990. Acorn size and geographical range in the North American oaks (Quercus L.) J. Biogr. 17, 327-332. https://doi.org/10.23 07/2845128

Aizen, M.A.; Woodcock, H.; 1992. Latitudinal trends in acorn size in eastern North American species of Quercus. Can. J. Bot. 70, 1218-1222. https://doi.org/10.1139/b92-153

Alejano, R.; Domínguez-Delmas, M.; García-González, I.; Wazny, T.; Vázquez-Piqué, J.; Fernández-Martínez, M.; 2019. The age of black pine (Pinus nigra Arn. ssp. salzmanni (Dunal) Franco) mother trees has no effect on seed germination and on offspring seedling performance. Ann. For. Sci. 76, 15. https://doi.org/10.1007/s13595-019-0801-7

Andrew, W.; Bartlow, A.W.; Agosta, S.J.; Curtis, R.; Yi, X.; Steele, M.A.; 2018. Acorn size and tolerance to seed predators: the multiple roles of acorns as food for seed predators, fruit for dispersal and fuel for growth. Integrative Zoology 13, 251-266. https://doi. org/10.1111/1749-4877.12287

Aranda, I.; Pardos, M.; Puértolas, J.; Jiménez, M.D.; Parods, J.A.; 2007. Water-use efficiency in cork oak (Quercus usber) is modified by the interaction of water and light availabilities. Tree Physiol 27, 671-677. https://doi.org/10.1093/treephys/27.5.671

Bartlow, A.W.; Agosta, S.; Curtis, R.; Yi, X.; Steele, M.A.; 2018. Acorn size and tolerance to seed predators: the multiple roles of acorns as food for seed predators, fruit for dispersal and fuel for growth. Integrative Zoology 13, 251-266. https://doi.org/10.1111/ $1749-4877.12287$

Calama, R.; Manso, R.; Lucas-Borja, M.E.; Espelta, J.M.; Piqué, M.; Bravo, F.; del Peso, C.; Pardos, M.; 2017. Natural regeneration in Iberian pines: a review of dynamic processes and proposals for management. For. Syst. 26(2). https://doi.org/10.5424/fs/2017262-11255

Carey, E.V.; Sala, A.; Keane, M.; Callaway. R.M.; 2001. Are old forests underestimated as global carbon sinks? Glob. Change Biol. 7, 339-344. https://doi.org/10.1046/j.1365-24 86.2001.00418.x 
Franklin, J.F.; Spies, T.A.; 1991. Ecological definitions of old-growth Douglas-fir forests. In: Wildlife and Vegetation of Unmanaged Douglas-Fir Forests. Report PNW-GTR285, Portland, USDA, For. Serv., 61-69.

Frelich, L.E.; Reich, P.B.; 2003. Perspectives on development of definitions and values related to old-growth forests. Environmental Reviews 11: S9-S22. https://doi.org/10.1139/ a03-011

García-Nogales, A.; Linares, J.C.; Laureano, R.G.; Seco, J.I.; Merino, J.; 2016. Rangewide variation in life_history phenotypes: spatiotemporal plasticity across the latitudinal gradient of the evergreen oak Quercus ilex. J. Biogeog. 43, 2366-2379. https://doi. org $/ 10.1111 /$ jbi.12849

Gómez, J.M.; 2004. Bigger is not always better: conflicting selective pressures on seed size in Quercus ilex. Evolution 58, 71-80. https://doi.org/10.1111/j.0014-3820.2004.tb01 574.X

Grove, AT.; Rackham, O.; 2001. The Nature of Mediterranean Europe: An Ecological History. Yale University Press, New Haven \& London.

Lawless, JF.; 2003. Statitical models and methods for lifetime data. John Wiley \& Sons inc. Hoboken. https://doi.org/10.1002/9781118033005

Lindenmayer, D.B.; Franklin, J.F.; 2002. Congruence between natural and human forest disturbance: a case study for Australian montane ash forest. For. Ecol. Managem. 155, 319 -335. https://doi.org/10.1016/S0378-1127(01)00569-2

Long, T.J.; Jones, R.H.; 1996. Seedling growth strategies and seed size effects in fourteen oak species native to different soil moisture habitats. Trees 11, 1-8. https://doi.org/10. $1007 / \mathrm{s} 004680050051$

Lucas-Borja, M.E.; Candel-Pérez, D.; Onkelinx, T.; Fule, P.Z.; Moya, D.; Gómez, R.; de las Heras, J.; 2017. Early Mediterranean pine recruitment in burned and unburned Pinus nigra Arn. ssp salzmannii stands of Central Spain: influence of species identity, provenances and post-dispersal predation. For. Ecol. Manag. 390, 203-211. https://doi.org/ 10.1016/j.foreco.2017.01.026

McCullagh, P.; Nelder, J.A.; (1989) Generalized Linear Models. 2nd Edition, Chapman and Hall, London. https://doi.org/10.1007/978-1-4899-3242-6

Marcelo, A.; Aizen, M.A.; Patterson W.A.; III, 1990. Acorn size and geographical range in the North American oaks (Quercus L.). J. Biogeogr. 17, 327-332. https://doi.org/10. $2307 / 2845128$

Merouani, H.; Branco, C.; Almeida, M.H.; Pereira, J.S.; 2001. Effects of acorn storage duration and parental tree on emergence and physiological status of Cork oak (Quercus suber L.) seedlings. Ann. For. Sci. 58, 543-554. https://doi.org/10.1051/forest:2001144

Moles, A.T.; Leishman, M.R.; 2008. The seedling as part of a plant's life history strategy. En: Seedling Ecology and Evolution (ed. M.A. Leck, V.T. Parker, R.L. Simpson), 217238. https://doi.org/10.1017/CBO9780511815133.012

Montero, G.; Cañellas, I.; 1999. Manual de reforestación y cultivo de alcornoque (Quercus suber L.). $\mathrm{M}^{\mathrm{o}}$ de Agricultura, Pesca y Alimentación. INIA, 103 p.

Mukassabi, T.A.; Polwart, A.; Coleshaw, T.; Thomas, P.A.; 2012. Scots pine seed dynamics on a waterlogged site. Trees 26: 1305-1315. https://doi.org/10.1007/s00468-012-0706-7

Nadrowski, K.; Wirth, C.; Scherer-Lorenzen, M.; 2010. Is forest diversity driving ecosystem function and service? Current Opinion in Environmental Sustainability 524, 75-79. https://doi.org/10.1016/j.cosust.2010.02.003 
Oliver, C.D.; Larson, B.C.; 1990. Forest stand dynamics. Biological resource management series. Mc Graw-Hill, USA

Peterken, G.F.; 1996. Natural woodland: ecology and conservation in northern temperate regions. Cambridge University Press

Petit, R.; Hampe, A.; 2006. Some evolutionary consequences of being a tree. Ann Rev Ecology, Evol and Systematics 37, 187-214. https://doi.org/10.1146/annurev.ecolsys. 37.091305.110215

Pillsbury, N.H.; Mc Donald, P.M.; 1990. Reliability of tanoak volume equations when applied to different areas, West. J. App. For. 10: 72-78. https://doi.org/10.1093/wjaf/ 10.2.72

Pons, J.; Pausas, J.G.; 2012. The coexistence of acorns with different maturation patterns explains acorn production variability in cork oak. Oecologia 169: 723-731. https://doi. org/10.1007/s00442-011-2244-1

Pukkala, T., Kolström, T., 1992. A stochastic spatial regeneration model for Pinus sylvestris. Scand. J. For. Res. 7, 377-385. https://doi.org/10.1080/02827589209382730

Sánchez-Montes de Oca, E.J.; Badano, E.I.; Silva-Alvarado, L.E.; Flores, J.; BarragánTorres, F.; Flores-Cano, J.A.; 2018. Acorn weight as determinant of germination in red and white oaks: evidences from a common-garden greenhouse experiment. Ann. For. Sci. 75, 12. https://doi.org/10.1007/s13595-018-0693-y

Shi, W.; Villar-Salvador, P.; Li, G.; Jiang, X.; 2019. Acorn size is more important than nursery fertilization for outplanting performance of Quercus variabilis container seedlings. Ann. For. Sci. 76, 22. https://doi.org/10.1007/s13595-018-0785-8

Smith, C.C.; Fretwell, S.D.; 1974. The optimal balance between size and number of offspring. The American Naturalist 109, 499-506. https://doi.org/10.1086/282929

Torres, M.; Frutos, G.; 1989. Analysis of germination curves of aged fennel seeds by mathematical models. Environ Exp. Bot. 29(3), 409-415. https://doi.org/10.1016/0098-84 72(89)90016-6

Vieira Natividade, J.; 1991. Subericultura. Mo de Agricultura, Pesca y Alimentación, 448p.

Westoby, M.; Jurado, J.; Leishman, M.; 1992. Comparative evolutionary ecology of seed size. Trends Ecol. Evol. 7, 368-372. https://doi.org/10.1016/0169-5347(92)90006-W

Wirth, C.; Messier, C.; Bergeron, Y.; Frank, D.; Fankhänel, A.; 2009. Old-Growth Forest Definitions: a Pragmatic View. En: Wirth Christian et al., ed. Old-Growth Forests. Berlin Heidelberg: Springer Verlag, 11-33. https://doi.org/10.1007/978-3-540-92706$8 \_2$

Xiao, Z.; Zhang, Z.; Wang, Y.; 2004. Dispersal and germination of big and small nuts of Quercus serrata in a subtropical broad-leaved evergreen forest. For. Ecol. Manage. 195, 141-150. https://doi.org/10.1016/j.foreco.2004.02.041

Yi, X.; Zhang, J.; Wang, Z.; 2015. Large and small acorns contribute equally to early-stage oak seedlings: a multiple species study. Eur. J. For. Res. 134, 1019-1026. https://doi. org/10.1007/s10342-015-0906-y 
